\title{
Satisfação dos Profissionais do Centro de Saúde São João (2007 e comparação com 2001 a 2006)
}

Alberto Augusto Oliveira Pinto Hespanhol*

\section{RESUMO}

Objectivos: Avaliar a satisfação global e a satisfação com os diferentes aspectos do exercício da profissão a nível de todos os elementos do Centro de Saúde São João (CSSJ).

Métodos: Um questionário auto-preenchido enviado pelo correio interno do CSSJ a todos os Profissionais que estavam ao serviço na semana de 21 a 25 de Maio de 2007.

Resultados: Enviámos 32 questionários e recebemos 31 (taxa de resposta de 97\%). A percentagem dos profissionais respondentes que declararam estar completamente satisfeitos, muito satisfeitos ou satisfeitos com o trabalho na sua globalidade é de $100 \%$, com excepção dos enfermeiros que é de $90 \%$.

Nas componentes da satisfação profissional que os profissionais declararam obter mais satisfação predominam os factores intrínsecos.

Por outro lado, as questões referidas como causadoras de maior insatisfação foram, nos médicos, a «Possibilidade de usar técnicas evoluídas»; nos enfermeiros, «A possibilidade de você se integrar na comunidade»; e, nos administrativos e auxiliares de apoio e vigilância, o «Vencimento», todos factores extrínsecos da satisfação profissional, com excepção nos enfermeiros, que é um factor intrínseco da satisfação profissional.

Discussão: A maioria dos profissionais parece estar satisfeita com o trabalho no CSSJ na sua globalidade. Nas componentes da satisfação profissional que os três estratos profissionais declararam provocar mais satisfação predominam os factores intrínsecos e nas componentes da satisfação profissional que declararam provocar mais insatisfação predominam os factores extrínsecos.

Palavras-Chave: Qualidade de Cuidados; Satisfação Profissional; Cuidados de Saúde Primários.

\section{INTRODUÇÃO}

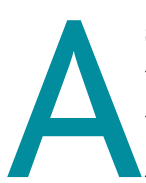

satisfação pode ser entendida como o resultado da avaliação que uma pessoa faz, em jeito de balanço, relativamente ao grau de realização dos seus valores, necessidades, preferências e expectativas, ou seja, é uma pessoa perceber ou sentir que aquilo que recebe está de acordo com aquilo que esperava obter. ${ }^{1}$

A satisfação profissional é um conjunto de diferentes facetas, algumas das quais provocam mais satisfação ou são mais aceitáveis do que outras. É influencia-

*Professor Associado com Agregação Convidado do Departamento de Clínica Geral da Faculdade de Medicina do Porto e Director do Centro de Saúde São João (Porto), onde também exerce as funções de Médico de Família da por forças internas e externas ao ambiente laboral, sofrendo alterações com o decorrer do tempo. Ela existe quando o trabalhador se apercebe que os benefícios do trabalho excedem os seus custos por uma margem suposta por ele como sendo adequada às circunstâncias. $^{2}$

A Qualidade em Saúde requer o cumprimento de um determinado número de requisitos e de interesses de grupos envolvidos, muitas vezes conflituosos. A perspectiva que tem sido menos frequentemente utilizada pelos investigadores no estudo da Qualidade dos serviços prestados pelos Centros de Saúde é a dos profissionais através da sua satisfação. ${ }^{1,-9}$

Herzberg preconizou, na sua Teoria da Higiene-mo- 
tivação, a existência de dois tipos de factores de trabalho:

- intrínsecos, factores de motivação que promovem a satisfação profissional, como por exemplo a realização de tarefas, o reconhecimento por essa realização, a natureza do próprio trabalho, a responsabilidade envolvida e o crescimento ou avanço proporcionado;

- extrínsecos, factores higiénicos que impedem a satisfação profissional, como por exemplo a política e administração da companhia, supervisão, relações interpessoais com o supervisor, colegas e subordinados, condições de trabalho, salário, estatuto e segurança. ${ }^{2,10,11}$

Em 1998 foi celebrado um Acordo de Cooperação entre a Administração Regional de Saúde do Norte e a Faculdade de Medicina da Universidade do Porto, designado por «Projecto Tubo de Ensaio», de que resultou, em 1999, a inauguração do Centro de Saúde São João (CSSJ). ${ }^{12-15}$ Desde 2001 temos vindo a elaborar anualmente inquéritos de satisfação aos profissionais do CSSJ. Nesse sentido, decidimos realizar uma investigação para avaliar a satisfação global e a satisfação com os diferentes aspectos do exercício da profissão a nível de todos os elementos do CSSJ.

\section{MÉTODOS}

Enviamos um questionário auto-preenchido pelo correio interno do CSSJ a todos os Médicos, Enfermeiros, Administrativos e Auxiliares de Apoio e de Vigilância que estavam ao serviço durante a semana de 21 a $25 \mathrm{de}$ Maio de 2007 ( $\mathrm{n}=32)$.

O questionário incluía um grupo de 21 questões sobre satisfação para os médicos e de 19 para os outros profissionais, as quais deviam ser respondidas com referência a uma escala do tipo Likert de 5 pontos. ${ }^{8,9}$

$\mathrm{Na}$ análise estatística realizámos uma tabela de frequência para todas as variáveis. Efectuámos análise das frequências através da estatística descritiva, utilizando frequências absolutas $\left(\mathrm{N}^{\circ}\right)$ e relativas (\%).

As variáveis de satisfação profissional foram apresentadas no questionário sob a forma de variáveis categóricas com cinco categorias $(1=$ muito insatisfeito, $2=$ insatisfeito, $3=$ satisfeito, $4=$ muito satisfeito, $5=$ completamente satisfeito), mas incluídas na tabelas de frequência sob a forma de variáveis categóricas com 2 categorias (Satisfeito $=3+4+5$; Insatisfeito $=1+2$ ). Recodificámos as categorias das diferentes variáveis de satisfação profissional devido ao facto de estas apresentarem uma grande assimetria de valores.

\section{RESULTADOS}

Dos 32 questionários auto-preenchidos enviados recebemos 31, o que corresponde a uma taxa de resposta de $97 \%$. Analisando por estrato profissional, foram enviados para os médicos 14 questionários e recebidos 13 , para os enfermeiros 10 e recebidos 10 e para os administrativos e auxiliares de apoio e vigilância 8 e recebidos 8 , a que correspondem taxas de resposta de, respectivamente, $93 \%, 100 \%$ e $100 \%$.

Nos médicos e nos administrativos e auxiliares de apoio e vigilância a percentagem de respondentes que declararam estar completamente satisfeitos, muito satisfeitos ou satisfeitos com o trabalho na sua globalidade é de $100 \%$, enquanto que nos enfermeiros é de $90 \%$ (Quadros I-III).

Das componentes da satisfação profissional que obtiveram 100\% de Médicos de Família declarando estar completamente satisfeitos, muito satisfeitos ou satisfeitos, e portanto provocando mais satisfação profissional, doze são factores intrínsecos e quatro são factores extrínsecos da satisfação profissional. Por outro lado, a questão referida como causadora de maior insatisfação foi a "Possibilidade de usar técnicas evoluídas» (85\%), um factor extrínseco à satisfação profissional (Quadro I).

Das componentes da satisfação profissional que obtiveram $100 \%$ de Enfermeiros declarando estar completamente satisfeitos, muito satisfeitos ou satisfeitos, e, portanto, provocando mais satisfação profissional, duas são factores intrínsecos e duas são factores extrínsecos da satisfação profissional. Por outro lado, a questão referida como causadora de maior insatisfação foi «A possibilidade de você se integrar na comunidade» (70\%), um factor intrínseco à satisfação profissional (Quadro I).

Das componentes da satisfação profissional que obtiveram $100 \%$ de Administrativos e Auxiliares de Apoio eVigilância declarando estar completamente satisfeitos, muito satisfeitos ou satisfeitos e, portanto, provocando mais satisfação profissional, oito são factores intrínsecos e quatro são factores extrínsecos da satisfa- 


\begin{tabular}{|c|c|c|c|c|c|c|c|}
\hline & ${ }^{*}(n=7)$ & ${ }^{* *}(n=9)$ & ${ }^{* * *}(n=8)$ & ${ }^{* * * *}(n=10)$ & ${ }^{* * * * *}(n=11)$ & ${ }^{* * * * * *}(n=14)$ & ${ }^{* * * * * * *}(n=13)$ \\
\hline 1. Ouvir os problemas dos utentes & 86 & 100 & 62 & 90 & 91 & 100 & 100 \\
\hline 2. Procurar conhecer os utentes e suas famílias & 86 & 100 & 62 & 90 & 100 & 100 & 100 \\
\hline $\begin{array}{l}\text { 3. Procurar ajudar os utentes e compreender } \\
\text { os seus próprios problemas }\end{array}$ & 100 & 100 & 75 & 90 & 91 & 100 & 100 \\
\hline $\begin{array}{l}\text { 4. Procurar que os utentes tomem as atitudes } \\
\text { correctas em relação à sua própria saúde }\end{array}$ & 100 & 89 & 100 & 100 & 91 & 100 & 100 \\
\hline 5. A possibilidade de você se integrar na comunidade & 71 & 89 & 100 & 100 & 100 & 93 & 100 \\
\hline 6. As possibilidades que tem para ajudar os utentes & 71 & 78 & 75 & 100 & 91 & 86 & 100 \\
\hline 7. Os seus conhecimentos técnico-profissionais & 100 & 89 & 100 & 100 & 100 & 93 & 100 \\
\hline 8. Liberdade de escolher o método de trabalho & 71 & 78 & 87 & 100 & 100 & 100 & 100 \\
\hline 9. Grau de responsabilidade que lhe é atribuída & 86 & 100 & 100 & 100 & 100 & 100 & 100 \\
\hline 10. Grau de variedade (monotonia no trabalho) & 71 & 78 & 100 & 100 & 100 & 100 & 100 \\
\hline 11. Relações de trabalho com colegas & 71 & 89 & 100 & 100 & 100 & 100 & 100 \\
\hline $\begin{array}{l}\text { 12. Oportunidade para demonstrar as suas } \\
\text { capacidades }\end{array}$ & 71 & 100 & 100 & 100 & 100 & 100 & 100 \\
\hline 13. Reconhecimento pela sua dedicação ao trabalho & 86 & 89 & 87 & 100 & 91 & 100 & 100 \\
\hline 14. Condições físicas do local de trabalho & 86 & 89 & 100 & 100 & 100 & 100 & 92 \\
\hline 15. Vencimento & 57 & 44 & 87 & 100 & 82 & 100 & 92 \\
\hline 16. Horas de trabalho & 86 & 89 & 87 & 100 & 82 & 93 & 92 \\
\hline 17. Possibilidade de usar técnicas evoluídas & 71 & 78 & 87 & 80 & 73 & 86 & 85 \\
\hline $\begin{array}{l}\text { 18. O modo como é administrada a sua } \\
\text { unidade de saúde }\end{array}$ & 71 & 100 & 87 & 100 & 91 & 100 & 100 \\
\hline 19. Liberdade de prescrição & 71 & 100 & 100 & 100 & 100 & 100 & 100 \\
\hline $\begin{array}{l}\text { 20. Capacidade para fazer diagnósticos } \\
\text { correctos e completos }\end{array}$ & 71 & 78 & 100 & 100 & 100 & 100 & 100 \\
\hline 21. Satisfação global no trabalho & 100 & 100 & 87 & 100 & 91 & 100 & 100 \\
\hline
\end{tabular}

Factor extrínseco

Factor intrínseco

ção profissional. Por outro lado, a questão referida como causadora de maior insatisfação foi o «Vencimento» (75\%), facto extrínseco à satisfação profissional (Quadro III).

\section{DISCUSSÃO}

Este questionário já tinha sido aplicado aos Médicos de Família, aos Enfermeiros e aos Administrativos e Auxiliares de Apoio e Vigilância do Centro de Saúde São João em 2001, 2002, 2003, 2004, 2005 e 2006.

A maioria dos profissionais parece estar satisfeita com o trabalho no CSSJ na sua globalidade, dado que a percentagem dos três estratos profissionais que de- clararam estar completamente satisfeitos, muito satisfeitos ou satisfeitos com o trabalho na sua globalidade foi em todos os estudos de $100 \%$, com excepção do estudo de 2003 em que foi de 87\% nos Médicos de Família e nos Enfermeiros, no estudo de 2005 em que foi de 91\% nos Médicos de Família e no estudo de 2007, em que foi de $90 \%$ nos Enfermeiros (Quadros I-III).

Os resultados obtidos no estudo actual, bem como na generalidade nos seis estudos anteriores, estão de acordo com a Teoria da Higiene-motivação sobre satisfação profissional, de Herzberg., ${ }^{2,10,11}$

Em todos os estudos, nas componentes da satisfação profissional que os três estratos profissionais declara- 


\begin{tabular}{|c|c|c|c|c|c|c|c|}
\hline \multicolumn{8}{|c|}{$\begin{array}{l}\text { QUADRO II. \% Enfermeiros que declararam estar completamente satisfeitos, muito satisfeitos ou satisfeitos a } \\
\text { nível do CS São João durante o ano de } 2001\left(^{*}\right), 2002(* *), 2003(* * *), 2004(* * * *), 2005(* * * *), 2006(* * * * *) \\
\text { e } 2007(* * * * * *)\end{array}$} \\
\hline & ${ }^{*}(n=12)$ & ${ }^{* *}(n=12)$ & ${ }^{* * *}(\mathrm{n}=8)$ & ${ }^{* * * *}(\mathrm{n}=8)$ & ${ }^{* * * * *}(\mathrm{n}=9)$ & ${ }^{* * * * * *}(\mathrm{n}=8)$ & ${ }^{* * * * * * *}(\mathrm{n}=10)$ \\
\hline 1. Ouvir os problemas dos utentes & 100 & 75 & 75 & 100 & 100 & 100 & 90 \\
\hline 2. Procurar conhecer os utentes e suas famílias & 67 & 75 & 75 & 87 & 100 & 88 & 80 \\
\hline $\begin{array}{l}\text { 3. Procurar ajudar os utentes e compreender } \\
\text { os seus próprios problemas }\end{array}$ & 92 & 75 & 75 & 100 & 100 & 100 & 80 \\
\hline $\begin{array}{l}\text { 4. Procurar que os utentes tomem as atitudes } \\
\text { correctas em relação à sua própria saúde }\end{array}$ & 75 & 92 & 87 & 87 & 100 & 100 & 90 \\
\hline 5. A possibilidade de você se integrar na comunidade & 67 & 67 & 75 & 100 & 89 & 75 & 70 \\
\hline 6. As possibilidades que tem para ajudar os utentes & 83 & 75 & 100 & 87 & 100 & 100 & 80 \\
\hline 7. Os seus conhecimentos técnico-profissionais & 92 & 100 & 100 & 87 & 100 & 100 & 90 \\
\hline 8. Liberdade de escolher o método de trabalho & 100 & 100 & 87 & 87 & 100 & 100 & 90 \\
\hline 9. Grau de responsabilidade que lhe é atribuída & 100 & 100 & 100 & 100 & 100 & 100 & 100 \\
\hline 10. Grau de variedade (monotonia no trabalho) & 75 & 92 & 87 & 75 & 100 & 100 & 100 \\
\hline 11. Relações de trabalho com colegas & 92 & 100 & 87 & 87 & 100 & 100 & 100 \\
\hline $\begin{array}{l}\text { 12. Oportunidade para demonstrar as suas } \\
\text { capacidades }\end{array}$ & 92 & 100 & 87 & 100 & 100 & 100 & 90 \\
\hline 13. Reconhecimento pela sua dedicação ao trabalho & 83 & 100 & 62 & 86 & 89 & 63 & 90 \\
\hline 14. Condições físicas do local de trabalho & 100 & 92 & 75 & 75 & 100 & 100 & 90 \\
\hline 15. Vencimento & 75 & 92 & 75 & 63 & 67 & 88 & 60 \\
\hline 16. Horas de trabalho & 100 & 92 & 100 & 100 & 100 & 100 & 90 \\
\hline 17. Possibilidade de usar técnicas evoluídas & 83 & 67 & 75 & 86 & 78 & 100 & 90 \\
\hline $\begin{array}{l}\text { 18. O modo como é administrada a sua unidade } \\
\text { de saúde }\end{array}$ & 92 & 92 & 87 & 86 & 100 & 100 & 100 \\
\hline \multicolumn{8}{|l|}{ 19. Liberdade de prescrição } \\
\hline \multicolumn{8}{|l|}{$\begin{array}{l}\text { 20. Capacidade para fazer diagnósticos correctos } \\
\text { e completos }\end{array}$} \\
\hline 21. Satisfação global no trabalho & 100 & 100 & 87 & 100 & 100 & 100 & 90 \\
\hline
\end{tabular}

Factor extrínseco

Factor intrínseco

ram provocar mais satisfação predominam os factores intrínsecos, com excepção do estudo de 2005 em que nos Administrativos e Auxiliares de Apoio e Vigilância predominam os factores extrínsecos (Quadros I-III).

Em todos os estudos, nas componentes da satisfação profissional que os Médicos de Família e os Administrativos e Auxiliares de Apoio e Vigilância declararam provocar mais insatisfação predominam os factores extrínsecos, com excepção do estudo de 2003 em que nos Médicos de Família predominam dois factores intrínsecos - «Ouvir os problemas dos utentes», «Procurar conhecer os utentes e suas famílias» - e do estudo de 2001 em que nos Administrativos e Auxiliares de Apoio e Vi- gilância predomina um factor intrínseco - «Reconhecimento pela sua dedicação ao trabalho» (Quadros I-III).

Também em todos os estudos, nas componentes da satisfação profissional que os Enfermeiros declararam provocar mais insatisfação predominam os factores extrínsecos, com excepção do estudo de 2001 em que nos Enfermeiros predominam dois factores intrínsecos «Procurar conhecer os utentes e suas famílias» e «A possibilidade de você se integrar na comunidade» - e nos estudos de 2003 e de 2006 um factor intrínseco - «Reconhecimento pela sua dedicação ao trabalho» (Quadros I-III).

Os resultados obtidos no estudo actual em relação 
QUADRO III. \% Administrativos e Auxiliares de Apoio e Vigilância que declararam estar completamente satisfeitos, muito satisfeitos ou satisfeitos a nível do CS São João durante o ano de $2001(*), 2002(* *), 2003(* * *), 2004(* * * *)$, $2005(* * * *), 2006(* * * * *)$ e $2007(* * * * * *)$

\begin{tabular}{|c|c|c|c|c|c|c|c|}
\hline & ${ }^{*}(\mathrm{n}=6)$ & ${ }^{* *}(\mathrm{n}=8)$ & ${ }^{* * *}(\mathrm{n}=8)$ & ${ }^{* * * *}(\mathrm{n}=8)$ & ${ }^{* * * * *}(\mathrm{n}=8)$ & ${ }^{* * * * * *}(\mathrm{n}=6)$ & ${ }^{* * * * * * *}(\mathrm{n}=8)$ \\
\hline 1. Ouvir os problemas dos utentes & 100 & 100 & 100 & 100 & 100 & 100 & 100 \\
\hline 2. Procurar conhecer os utentes e suas famílias & 83 & 100 & 100 & 100 & 100 & 100 & 100 \\
\hline $\begin{array}{l}\text { 3. Procurar ajudar os utentes e compreender os } \\
\text { seus próprios problemas }\end{array}$ & 100 & 88 & 100 & 100 & 88 & 100 & 100 \\
\hline $\begin{array}{l}\text { 4. Procurar que os utentes tomem as atitudes } \\
\text { correctas em relação à sua própria saúde }\end{array}$ & 100 & 75 & 100 & 100 & 100 & 100 & 100 \\
\hline 5. A possibilidade de você se integrar na comunidade & 83 & 88 & 100 & 100 & 100 & 100 & 100 \\
\hline 6. As possibilidades que tem para ajudar os utentes & 100 & 75 & 87 & 87 & 88 & 100 & 88 \\
\hline 7. Os seus conhecimentos técnico-profissionais & 83 & 88 & 100 & 100 & 88 & 100 & 88 \\
\hline 8. Liberdade de escolher o método de trabalho & 33 & 88 & 100 & 100 & 100 & 100 & 88 \\
\hline 9. Grau de responsabilidade que lhe é atribuída & 100 & 88 & 100 & 87 & 88 & 100 & 100 \\
\hline 10. Grau de variedade (monotonia no trabalho) & 100 & 75 & 100 & 100 & 88 & 100 & 88 \\
\hline 11. Relações de trabalho com colegas & 83 & 100 & 100 & 100 & 100 & 100 & 100 \\
\hline $\begin{array}{l}\text { 12. Oportunidade para demonstrar as suas } \\
\text { capacidades }\end{array}$ & 83 & 88 & 87 & 100 & 88 & 100 & 100 \\
\hline 13. Reconhecimento pela sua dedicação ao trabalho & 50 & 75 & 62 & 63 & 75 & 100 & 100 \\
\hline 14. Condições físicas do local de trabalho & 83 & 88 & 100 & 100 & 100 & 100 & 100 \\
\hline 15. Vencimento & 83 & 63 & 25 & 63 & 63 & 100 & 75 \\
\hline 16. Horas de trabalho & 83 & 100 & 100 & 100 & 100 & 100 & 100 \\
\hline 17. Possibilidade de usar técnicas evoluídas & 100 & 88 & 87 & 87 & 100 & 100 & 88 \\
\hline $\begin{array}{l}\text { 18. O modo como é administrada a sua unidade } \\
\text { de saúde }\end{array}$ & 100 & 100 & 100 & 100 & 100 & 100 & 100 \\
\hline \multicolumn{8}{|l|}{ 19. Liberdade de prescrição } \\
\hline \multicolumn{8}{|l|}{$\begin{array}{l}\text { 20. Capacidade para fazer diagnósticos correctos } \\
\text { e completos }\end{array}$} \\
\hline 21. Satisfação global no trabalho & 100 & 100 & 100 & 100 & 100 & 100 & 100 \\
\hline
\end{tabular}

Factor extrínseco

Factor intrínseco

aos Médicos de Família estão de acordo com os trabalhos que estudaram a satisfação profissional a nível desses clínicos, em que aparece uma predominância dos factores intrínsecos do trabalho nas componentes que provocam mais satisfação profissional e uma predominância dos factores extrínsecos do trabalho nas componentes que provocam mais insatisfação profissional. ${ }^{3-7}$

\section{CONCLUSÕES}

- As componentes da satisfação que os profissionais do CSSJ declararam provocar mais satisfação profissional são na sua generalidade factores intrínsecos do trabalho.
- As componentes da satisfação que os profissionais do CSSJ declararam provocar mais insatisfação profissional são na sua generalidade factores extrínsecos do trabalho.

- Ao longo de 2001 a 2007 verifica-se uma certa estabilidade no padrão da satisfação dos profissionais do CSSJ.

\section{REFERÊNCIAS BIBLIOGRÁFICAS}

1. Graça L. Instrumentos para a melhoria contínua da qualidade: a satisfação profissional dos profissionais de saúde nos Centros de Saúde. Lisboa: Direcção Geral da Saúde, Sub-Direcção Geral para a Qualidade; 1999.

2. Fraser TM. Human stress, work and job satisfaction: a critical appro- 
ach. 2nd ed. Geneva: International Labour Office; 1984.

3. Branthwaite A, Ross A. Satisfaction and job stress in general practice. Fam Pract 1988 Jun; 5 (2): 83-93.

4. Cooper CL, Rout U, Faragher B. Mental health, job satisfaction, and job stress among general practitioners. BMJ 1989 Feb 11; 298 (6670): 366-70 .

5. Sutherland VJ, Cooper CL. Job stress, satisfaction, and mental health among general practitioners before and after introduction of new contract. BMJ 1992 Jun 13; 304 (6841): 1545-8.

6. Pires B, Cerdeira F. Satisfação profissional de clínicos gerais num centro de saúde. Rev Port Clin Geral 1989 Jan-Fev; 6 (1): 6-13.

7. Vieira D, Viegas I. Satisfação profissional do clínico geral. Rev Port Clin Geral 1991; 8 (7): 210-5.

8. Hespanhol A, Pereira A, Sousa Pinto A. Satisfacción laboral en los médicos portugueses de medicina general. Aten Primaria 1999 Nov 15; 24 (8): 456-61.

9. Hespanhol A, Pereira A, Sousa Pinto A. Insatisfação profissional em medicina geral e familiar: um problema intrínseco dos médicos ou das condições de trabalho. Rev Port Clin Geral 2000 Maio-Jun; 16 (3): 183-99.

10. Hersey P, Blanchard K H. Management of organizational behavior - utilizing human resources. 6th ed. New Jersey: Prentice-Hall Internacional; 1993.
11. Santos GG. Teorias da motivação em contexto organizacional. Braga: Mestrado em Gestão de Recursos Humanos, Escola Economia e Gestão da Universidade do Minho; 1999.

12. Hespanhol A. O Projecto Tubo de Ensaio. Cad Aten Primaria 1999; 6 (2): 125.

13. Hespanhol A, Malheiro A, Pinto AS. O Projecto «Tubo de Ensaio» - Breve história do Centro de Saúde S. João. Rev Port Clin Geral 2002 MaioJun; 18 (3): 171-86.

14. Hespanhol A. Ensaio de um novo modelo de organização no Centro Saúde São João do Porto. Brotéria 2004 Dez; 159: 513-9.

15. Hespanhol A, Pinto AS. Cinco anos do Centro de Saúde São João «Tubo de Ensaio». Arq Medicina 2005 Maio, 19 (3): 103-11.

\section{ENDEREÇO PARA CORRESPONDÊNCIA}

Alberto Pinto Hespanhol

Rua Professor Mota Pinto, $197-2^{\circ}$ Esq.

4100 Porto

Recebido em 26/01/08

Aceite para publicação em 16/11/08

\section{ABSTRACT}

Aims: To evaluate the overall satisfaction of São João Health Center's professionals and their satisfaction with all other aspects of the service provided by the Health Center.

Methods: A self administered questionnaire distributed by the Health Center's internal mail to all the professionals that were on duty during the week from 21th until 25th May 2007.

Results: We sent 32 questionnaires and we received 31 (response rate of 97\%). The percentage of the respondent professionals that declared to be completely satisfied, very satisfied or satisfied with the overall job satisfaction is $100 \%$, with the exception of the nurses which is $90 \%$.

In the dimensions of job satisfaction that the professionals declared to obtain more job satisfaction the intrinsic factors predominate.

On the other hand, the questions refered as provoking more job dissatisfaction were in the Family Doctors the «Difficulties in using developed skills», in the nurses the "The possibility of your integration in the community» and in the administrative and auxiliary staff the "rate of pay», all extrinsic job factors, with the exception in the nurses which is a intrinsic job factor. Discussion: The majority of the professionals seems to be satisfied with the overall work in São João Health Center's. In the dimensions of job satisfaction that the three kinds of professionals declared to obtain more job satisfaction the intrinsic factors predominate and in the dimensions of job satisfaction that they declared to obtain more job dissatisfaction the extrinsic factors predominate.

Keywords: Quality of Health Care; Job Satisfaction; Primary Health Care. 